\title{
Article
}

\section{Heritability of Type 2 Diabetes in the Washington State Twin Registry}

\author{
Ally R. Avery and Glen E. Duncan \\ Department of Nutrition and Exercise Physiology, Washington State University, Spokane, WA, USA
}

\begin{abstract}
Approximately $12 \%$ of U.S. adults have type 2 diabetes (T2D). Diagnosed T2D is caused by a combination of genetic and environmental factors including age and lifestyle. In adults 45 years and older, the Discordant Twin (DISCOTWIN) consortium of twin registries from Europe and Australia showed a moderate-to-high contribution of genetic factors of T2D with a pooled heritability of $72 \%$. The purpose of this study was to investigate the contributions of genetic and environmental factors of T2D in twins 45 years and older in a U.S. twin cohort (Washington State Twin Registry, WSTR) and compare the estimates to the DISCOTWIN consortium. We also compared these estimates with twins under the age of 45. Data were obtained from 2692 monozygotic (MZ) and same-sex dizygotic (DZ) twin pairs over 45 and 4217 twin pairs under 45 who responded to the question 'Has a doctor ever diagnosed you with (type 2) diabetes?' Twin similarity was analyzed using both tetrachoric correlations and structural equation modeling. Overall, $9.4 \%$ of $\mathrm{MZ}$ and $14.7 \%$ of DZ twins over the age of 45 were discordant for T2D in the WSTR, compared to $5.1 \%$ of MZ and $8 \%$ of DZ twins in the DISCOTWIN consortium. Unlike the DISCOTWIN consortium in which heritability was $72 \%$, heritability was only $52 \%$ in the WSTR. In twins under the age of 45 , heritability did not contribute to the variance in T2D. In a U.S. sample of adult twins, environmental factors appear to be increasingly important in the development of T2D.
\end{abstract}

Keywords: body mass index; obesity; twins; type 2 diabetes

(Received 20 November 2018; accepted 25 February 2019)

Type 2 diabetes (T2D) is a complex disease that results from an interaction of genetic and environmental factors (Cornelis \& $\mathrm{Hu}, 2012$; Ribel-Madsen et al., 2012). Once considered a disease of older age, it is now prevalent in younger age groups, due in part to poor lifestyle factors such as a decrease in physical activity and an increase in energy-dense food consumption leading to higher rates of obesity (Dendup et al., 2018; Kolb \& Martin, 2017; McGavock et al., 2015; Pulgaron \& Delamater, 2014; Qi et al., 2008). Individuals with T2D are more likely to have comorbid conditions such as hypertension and hyperlipidemia (Lin et al., 2015), leading to an increase in healthcare costs. An estimated $12.2 \%$ of U.S. adults (Centers for Disease Control and Prevention, 2017) have diabetes. Healthcare expenditures for diabetes in the United States increased 26\% between 2012 and 2017 to an estimated \$327 billion dollars per year (American Diabetes Association, 2018). Given the increasing prevalence and substantial health burden of T2D, investigating the causal factors contributing to the disease remains an important topic for the scientific community.

Twin studies have shown a large genetic contribution to T2D (Carlsson et al., 2013; Condon et al., 2008; Poulsen et al., 2009). Recently, the Discordant Twin (DISCOTWIN) consortium examined the contribution of genetics and environment in 34,000 twin pairs over the age of 45 from seven twin registries in Europe and one in Australia and found a pooled heritability estimate of $72 \%$ (Willemsen et al., 2015). Using the comparative prevalence for

Author for correspondence: Ally R. Avery, Email: ally.avery@wsu.edu

Cite this article: Avery AR, Duncan GE. (2019) Heritability of Type 2 Diabetes in the Washington State Twin Registry. Twin Research and Human Genetics 22: 95-98, https:// doi.org/10.1017/thg.2019.11 countries provided by a report of the International Diabetes Federation, which is calculated by assuming that every country and region has the same age profile, the prevalence of $\mathrm{T} 2 \mathrm{D}$ in the United States (10.8) is nearly twice the average of the prevalence for the eight countries represented in the DISCOTWIN consortium (5.5) (International Diabetes Federation, 2017). Elucidating the reasons for this large discrepancy may contribute to better preventative and treatment efforts in the United States.

The goal of this article was to investigate the contributions of genetic and environmental factors to the variance of T2D in twins 45 years and older in an active U.S. twin cohort and compare the estimates with those reported in the DISCOTWIN consortium. We also compared the variance of estimates from the over 45 twins with those under 45. Given differences in environmental and lifestyle factors between the U.S. and European nations and Australia (Carlson, 2018; Díez et al., 2016; Semyonov et al., 2013), we hypothesized that (1) heritability would be lower and the variance attributable to the common and unique environment in T2D would be higher among U.S. twins over the age of 45 compared to those in the DISCOTWIN consortium, and (2) variance attributed to the common and unique environment would be higher in twins under the age of 45 compared to twins over 45 .

\section{Methods}

\section{Sample}

The Washington State Twin Registry (WSTR) is a communitybased registry of twin pairs primarily recruited through Washington State Department of Licensing (DOL) records (Afari et al., 2006; Strachan et al., 2013). The WSTR enrolls new 
pairs on a regular basis. All twins complete a questionnaire to enroll, which includes questions about health history, lifestyle behaviors and sociodemographic factors. To date, 9107 adult twin pairs have been enrolled in the Registry. Since 2006, we have collected follow-up data every 2-3 years with more detailed surveys. Standard questions about childhood similarity were used to classify twins as identical (monozygotic, MZ) or fraternal (dizygotic, DZ). When compared to DNA-based methods, these questions correctly determine zygosity with greater than 90\% accuracy (Jackson et al., 2001).

\section{Measures}

The WSTR enrollment survey has asked about a doctor's diagnosis of diabetes since 2002, but up until 2013 it did not specify type. All follow-up surveys have contained a question that asked specifically about a doctor's diagnosis of T2D. In the WSTR, 90\% of twins who answered the diabetes question at enrollment without mention of type reported type 2 in subsequent surveys. Thus, to increase the sample size, and given that $90-95 \%$ of all diabetes diagnoses are T2D (Qi et al., 2008), we included twin pairs who answered the nonspecific diabetes question (from earlier enrollment surveys) in addition to the pairs who answered the question that specified type (from later enrollment and all follow-up surveys). To ensure that we used data from the same wave of data collection, we excluded twin pairs with survey dates more than 2 years apart. Twin pairs where one or both twins were missing a response to the diabetes question were excluded. For twin pairs with more than one time point, the most recent survey completed was used.

\section{Statistical Analyses}

All analyses were conducted using R version 3.5.1 (R Core Team, 2017). Selected demographic characteristics were summarized by means for continuous variables and percentages for categorical variables. Within-pair tetrachoric correlations of T2D with $95 \%$ confidence intervals (CI) were computed for both $\mathrm{MZ}$ and $\mathrm{DZ}$ twins. We constructed structural equation models with 95\% CI using the OpenMx package (Neale et al., 2016) to estimate the variance in $\mathrm{T} 2 \mathrm{D}$ due to additive genetic $(A)$, shared environment $(C)$ and unique environment $(E)$ factors.

\section{Results}

We excluded 1985 opposite-sex twin pairs and 213 twin pairs in which one or both twins were missing an answer to the diabetes question, for a final pair $N$ of 6909 . We split the data by age (older or younger than 45), with 2692 pairs over 45 and 4217 pairs under 45. Descriptive statistics are summarized by zygosity and age group in Table 1. Overall, the WSTR sample had a mean age of 41 years, was $36 \%$ male, $86 \%$ non-Hispanic White and had a mean BMI of 25.9. Table 2 summarizes the proportion of within-pair T2D concordance and discordance by zygosity and age group.

Table 3 summarizes the within-pair correlations and estimates of variance in the WSTR by age group. The within-pair correlation for $\mathrm{MZ}$ twins over the age of 45 was higher $\left(r_{\mathrm{MZ}}=.76,95 \% \mathrm{CI}\right.$ $[0.69,0.81])$ than in DZ twins $\left(r_{\mathrm{DZ}}=.51,95 \%\right.$ CI $\left.[0.38,0.62]\right)$, suggesting a genetic influence on developing T2D. The correlation for MZ twins was not significantly different from the correlation reported by the DISCOTWIN consortium, but the correlation for DZ twins was significantly higher than the pooled correlation reported by DISCOTWIN $(.41,95 \%$ CI $[0.37,0.45])$. In the full ACE model, heritability contributed $52 \%$ of the variance, with
Table 1. Select demographic characteristics of twin pairs from the WSTR

\begin{tabular}{lcccc} 
& $\begin{array}{c}\text { MZ over } \\
45\end{array}$ & $\begin{array}{c}\text { DZ over } \\
45\end{array}$ & $\begin{array}{c}\text { MZ under } \\
45\end{array}$ & $\begin{array}{c}\text { DZ under } \\
45\end{array}$ \\
\cline { 2 - 5 } Pair N & 1682 & 1010 & 2922 & 1295 \\
\hline Mean age (SD) & $60.6(10.4)$ & $61.4(10.1)$ & $28.7(7.9)$ & $28.1(8.0)$ \\
\hline Male (\%) & 36.3 & 35.3 & 35.1 & 35.9 \\
\hline White (\%) & 90.8 & 93.6 & 81.0 & 85.2 \\
\hline Mean BMI (SD) & $27.3(5.8)$ & $27.2(5.7)$ & $24.8(5.2)$ & $25.3(5.8)$ \\
\hline T2D prevalence (\%) & 9.6 & 11.2 & 1.1 & 1.5 \\
\hline
\end{tabular}

Table 2. Number and percentage of twin-pair concordant and discordant for T2D

\begin{tabular}{|c|c|c|c|c|c|}
\hline & \multirow[b]{2}{*}{ Pair $N$} & \multicolumn{2}{|c|}{$\begin{array}{c}\text { Concordant } \\
\text { yes }\end{array}$} & \multicolumn{2}{|c|}{ Discordant } \\
\hline & & $N$ & $\%$ & $N$ & $\%$ \\
\hline MZ over 45 & 1682 & 83 & 4.9 & 158 & 9.4 \\
\hline DZ over 45 & 1010 & 39 & 3.9 & 148 & 14.7 \\
\hline MZ under 45 & 2922 & 8 & 0.3 & 48 & 1.6 \\
\hline DZ under 45 & 1295 & 4 & 0.3 & 30 & 2.3 \\
\hline
\end{tabular}

shared and unique environment each contributing 24\%. Shared environment was not significant, as the $95 \%$ CI contained zero. However, dropping $C$ did not result in an improvement in model fit. We examined sex differences and found no significant difference when parameters were constrained to be equal $(p=.36)$.

Next, we examined correlations and ACE estimates for twins under 45. The within-pair correlation for MZ twins was slightly higher $\left(r_{\mathrm{MZ}}=.68,95 \% \mathrm{CI}[0.49,0.81]\right)$ than the correlation for DZ twins $\left(r_{\mathrm{DZ}}=.60,95 \% \mathrm{CI}[0.31,0.79]\right)$. The best-fitting model did not include heritability, with shared environment contributing $65 \%$ of the variance and unique environment contributing $35 \%$. Again, we examined sex differences and found no significant difference when parameters were constrained to be equal $(p=.43)$. While the best-fitting model for twins under 45 included only $C$ and $E$, when we constrained the full ACE model parameters to be equal for twins over 45 and under 45 , we found no significant difference $(p=.77)$.

Finally, we examined the shared variance between BMI and $\mathrm{T} 2 \mathrm{D}$, as the increase in T2D prevalence has been associated with an increase in obesity. The bivariate path models are shown in Figure 1 . In twins over $45,34 \%$ of the variance in heritability and $5 \%$ of the variance in unique environment was shared by T2D and BMI. In twins under $45,27 \%$ of the variance in shared environment and $9 \%$ of the variance in unique environment was shared by $\mathrm{T} 2 \mathrm{D}$ and BMI.

\section{Discussion}

In this study, we found that the heritability of T2D in a sample of U.S. twins over the age of 45 is substantially lower than that found in a large sample of twins from several European and Australian twin cohorts. About half of the variance in T2D in the WSTR sample is attributable to genetic variance, with the remaining attributable in equal proportions to shared and nonshared environmental variance. In contrast, six out of nine of the DISCOTWIN cohorts 
Table 3. Twin correlations and the estimates of variance for $A, C$ and $E$

\begin{tabular}{|c|c|c|c|c|c|c|c|c|}
\hline \multirow[b]{2}{*}{ Age } & \multicolumn{2}{|c|}{ Twin correlations } & \multicolumn{3}{|c|}{ Estimates of variance components ${ }^{a}$} & \multirow[b]{2}{*}{$-2 \mathrm{LL}$} & \multirow[b]{2}{*}{$\mathrm{AIC}^{\mathrm{b}}$} & \multirow[b]{2}{*}{$p$} \\
\hline & MZ & DZ & $A$ & C & $E$ & & & \\
\hline \multirow[t]{3}{*}{ Over 45} & $0.76[0.69,0.81]$ & $0.51[0.38,0.62]$ & $0.52[0.26,0.80]$ & $0.24[0.01,0.48]$ & $0.24[0.18,0.31]$ & - & - & - \\
\hline & & & $0.78[0.71,0.83]$ & - & $0.22[0.17,0.29]$ & 3.4 & 1.4 & .07 \\
\hline & & & - & $0.67[0.61,0.73]$ & $0.33[0.27,0.40]$ & 15.7 & 13.7 & $<.001$ \\
\hline \multirow[t]{3}{*}{ Under 45} & $0.68[0.49,0.81]$ & $0.60[0.31,0.79]$ & $0.22[-0.32,0.83]$ & $0.47[-0.09,0.91]$ & $0.31[0.18,0.49]$ & - & - & - \\
\hline & & & $0.72[0.56,0.84]$ & - & $0.28[0.16,0.44]$ & 2.8 & 0.77 & .10 \\
\hline & & & - & $0.65[0.50,0.77]$ & $0.35[0.23,0.50]$ & 0.6 & -1.39 & .44 \\
\hline
\end{tabular}

Note: The best-fitting and most parsimonious models are shown in bold. $95 \% \mathrm{Cl}$ shown in square brackets.

aproportion of variance due to additive genetics $(A)$, common environment $(C)$ and unique environment $(E)$.

${ }^{b}$ Akaike's information criterion (AIC) is a global measure of goodness of fit, with negative values indicating a better fit when compared to the full ACE model.
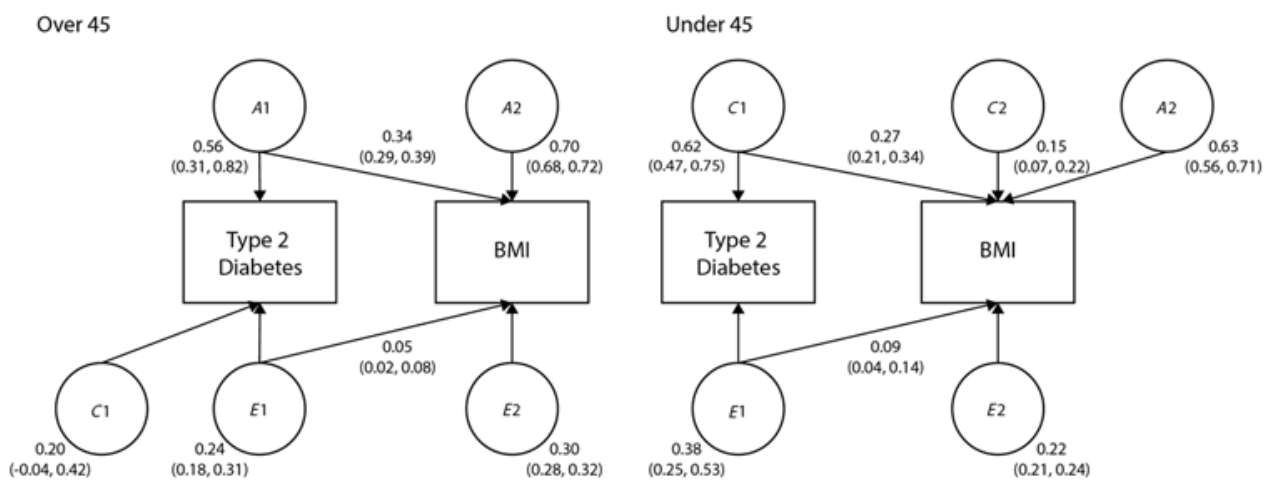

Fig. 1. Bivariate models showing the standardized path coefficients for additive genetic, shared and unique environmental influences on BMI and T2D in twins over 45 and under 45. Only one twin shown for clarity.

found that genetics explained well over half of the variance in T2D (64-79\%). This paper also showed that both shared and unique environment plays a large role in the development of T2D in adults under the age of 45 , with $65 \%$ of the variance in T2D attributable to the shared environment. Thus, the findings support our hypotheses that genetic factors explain a smaller proportion and environmental factors explain a larger proportion of the variance in T2D among twin adults residing in the United States than twins from European and Australian cohorts.

Little research has been done exploring the contributions of genetics and environment on twins with T2D in the United States. Newman et al. (1987) found that genetic factors were important in the development of T2D in a small sample of White male twins born between 1917 and 1927 identified by military records. The estimated genetic contribution using the proband concordance rate was $58 \%$, which is close to the estimate (52\%) calculated in our ACE model. Potential environmental or behavioral factors contributing to glucose tolerance were measured only in discordant MZ twins, and no significant pairwise differences were found.

One of the purposes of the DISCOTWIN consortium was to identify the number of T2D discordant MZ pairs who might be available for in-depth studies to elucidate the epigenetic mechanisms and causal environmental factors in disease development and progression. Across DISCOTWIN cohorts, except for the Longitudinal study of aging Danish twins (LSADT) (10.5\%) and Murcia twin registry (MTR) cohorts (12.3\%), the prevalence of T2D was lower (2.6-9.6\%) than in our WSTR sample of twins over 45 (10.4\%). Not surprisingly, there were fewer discordant MZ twins across the DISCOTWIN consortium (720 pairs, 5.1\%) compared to the WSTR sample of twins over 45 (158 pairs, 9.4\%). These discordant pairs are available for pooling with other U.S. and international twin registries to investigate the pathophysiology of T2D and better elucidate how genetic and environmental factors differ between regions.

\section{Limitations}

The current study has some limitations. Our data are from self-reports and were not corroborated with further testing, which could result in measurement bias. To increase the sample size, we included data from our enrollment survey that did not clarify diabetes type; $2 \%$ of twins reporting diabetes in our final sample completed only the enrollment survey. While this does contribute some measurement bias, it is likely a small problem as $90 \%$ of diabetes cases in the WSTR are T2D. Finally, our sample of WSTR twins is mostly White and of average to higher socioeconomic status. T2D prevalence is much higher among underserved populations in the United States such as non-Hispanic Black and Hispanic individuals (Geiss et al., 2014), and thus our results may not be generalizable to the larger U.S. population.

\section{Conclusion}

In contrast to twins from European and Australian cohorts, we found a lower contribution of genetics to the variation in T2D, and a modest contribution from unique environment in twins over 45. We found that genetics did not contribute to the variation in T2D in twins under 45 . Our findings suggest that differences in environmental factors between U.S. and other populations may 
differentially impact the development and progression of T2D, such as sedentary lifestyles and poor habitual diets that characterize most U.S. adults. Further research should explore the role of environmental factors to better understand the development, progression and treatment of T2D.

Acknowledgments. Siny Tsang was invaluable in helping with the interpretation of the findings. We thank the twin members of the Washington State Twin Registry for their participation in our research.

Financial support. This work was supported by a grant from the National Institute of Health [grant number R56AG042176].

\section{Conflict of interest. None.}

Ethical standards. The authors assert that all procedures contributing to this work comply with the ethical standards of the relevant national and institutional committees on human experimentation and with the Helsinki Declaration of 1975, as revised in 2008 .

\section{References}

Afari, N., Noonan, C., Goldberg, J., Edwards, K., Gadepalli, K., Osterman, B., ... Buchwald, D. (2006). University of Washington Twin Registry: Construction and characteristics of a community-based twin registry. Twin Research and Human Genetics, 9, 1023-1029.

American Diabetes Association. (2018). Economic costs of diabetes in the U.S. in 2017. Diabetes Care, 41, 917-928.

Carlson, M. (2018). Families unequal: Socioeconomic gradients in family patterns across the United States and Europe. In N. R. Cahn, J. Carbone, L. Fields DeRose, \& W. B. Wilcox (Eds.), Unequal family lives: Causes and consequences in Europe and the Americas (pp. 21-39). Cambridge, UK: Cambridge University Press.

Carlsson, S., Ahlbom, A., Lichtenstein, P., \& Andersson, T. (2013). Shared genetic influence of BMI, physical activity and type 2 diabetes: A twin study. Diabetologia, 56, 1031-1035.

Centers for Disease Control and Prevention. (2017). National diabetes statistics report, 2017. Retrieved from Atlanta, GA: Author.

Condon, J., Shaw, J. E., Luciano, M., Kyvik, K. O., Martin, N. G., \& Duffy, D. L. (2008). A study of diabetes mellitus within a large sample of Australian twins. Twin Research and Human Genetics, 11, 28-40.

Cornelis, M. C., \& Hu, F. B. (2012). Gene-environment interactions in the development of type 2 diabetes: Recent progress and continuing challenges. Annual Review of Nutrition, 32, 245-259.

Dendup, T., Feng, X., Clingan, S., \& Astell-Burt, T. (2018). Environmental risk factors for developing type 2 diabetes mellitus: A systematic review. International Journal of Environmental Research and Public Health, 15, E78.

Díez, J., Bilal, U., Cebrecos, A., Buczynski, A., Lawrence, R. S., Glass, T., .. Franco, M. (2016). Understanding differences in the local food environment across countries: A case study in Madrid (Spain) and Baltimore (USA). Preventive Medicine, 89, 237-244.
Geiss, L. S., Wang, J., Cheng, Y. J., Thompson, T. J., Barker, L., Li, Y., . . Gregg, E. W. (2014). Prevalence and incidence trends for diagnosed diabetes among adults aged 20 to 79 years, United States, 1980-2012. JAMA, 312, $1218-1226$.

International Diabetes Federation. (2017). IDF diabetes atlas (8th ed.). Brussels, Belgium: Author.

Jackson, R. W., Snieder, H., Davis, H., \& Treiber, F. A. (2001). Determination of twin zygosity: A comparison of DNA with various questionnaire indices. Twin Research, 4, 12-18.

Kolb, H., \& Martin, S. (2017). Environmental/lifestyle factors in the pathogenesis and prevention of type 2 diabetes. BMC Medicine, 15, 131.

Lin, P. J., Kent, D. M., Winn, A., Cohen, J. T., \& Neumann, P. J. (2015). Multiple chronic conditions in type 2 diabetes mellitus: Prevalence and consequences. American Journal of Managed Care, 21, e23-34.

McGavock, J., Dart, A., \& Wicklow, B. (2015). Lifestyle therapy for the treatment of youth with type 2 diabetes. Current Diabetes Reports, 15, 568.

Neale, M. C., Hunter, M. D., Pritikin, J. N., Zahery, M., Brick, T. R., Kirkpatrick, R. M., . . Boker, S. M. (2016). OpenMx 2.0: Extended structural equation and statistical modeling. Psychometrika, 81, 535-549.

Newman, B., Selby, J. V., King, M. C., Slemenda, C., Fabsitz, R., \& Friedman, G. D. (1987). Concordance for type 2 (non-insulin-dependent) diabetes mellitus in male twins. Diabetologia, 30, 763-768.

Poulsen, P., Grunnet, L. G., Pilgaard, K., Storgaard, H., Alibegovic, A., Sonne, M. P., ... Vaag, A. (2009). Increased risk of type 2 diabetes in elderly twins. Diabetes, 58, 1350-1355.

Pulgaron, E. R., \& Delamater, A. M. (2014). Obesity and type 2 diabetes in children: Epidemiology and treatment. Current Diabetes Reports, 14, 508. doi: 10.1007/s11892-014-0508-y

Qi, L., Hu, F. B., \& Hu, G. (2008). Genes, environment, and interactions in prevention of type 2 diabetes: A focus on physical activity and lifestyle changes. Current Molecular Medicine, 8, 519-532.

R Core Team. (2017). $R$ : A language and environment for statistical computing (Version 3.5.1). Vienna, Austria: R Foundation for Statistical Computing. Retrieved from https://www.R-project.org/

Ribel-Madsen, R., Fraga, M. F., Jacobsen, S., Bork-Jensen, J., Lara, E., Calvanese, V., ... Poulsen, P. (2012). Genome-wide analysis of DNA methylation differences in muscle and fat from monozygotic twins discordant for type 2 diabetes. PLoS One, 7, e51302.

Semyonov, M., Lewin-Epstein, N., \& Maskileyson, D. (2013). Where wealth matters more for health: The wealth-health gradient in 16 countries. Social Science \& Medicine, 81, 10-17.

Strachan, E., Hunt, C., Afari, N., Duncan, G., Noonan, C., Schur, E., ... Buchwald, D. (2013). University of Washington Twin Registry: Poised for the next generation of twin research. Twin Research and Human Genetics, 16, 455-462. doi: 10.1017/thg.2012.124

Willemsen, G., Ward, K. J., Bell, C. G., Christensen, K., Bowden, J., Dalgard, C., ... Spector, T. (2015). The concordance and heritability of type 2 diabetes in 34, 166 twin pairs from international twin registers: The Discordant Twin (DISCOTWIN) Consortium. Twin Research and Human Genetics, 18, 762-771. 